\title{
Can Square Kilometre Array phase 1 go much beyond the LHC in supersymmetry search?
}

\author{
Arpan Kar, ${ }^{1}$ Sourav Mitra, ${ }^{2}$ Biswarup Mukhopadhyaya, ${ }^{1}$ and Tirthankar Roy Choudhury ${ }^{3}$ \\ ${ }^{1}$ Regional Centre for Accelerator-based Particle Physics, Harish-Chandra Research Institute, \\ HBNI, Chhatnag Road, Jhunsi, Allahabad 211 019, India \\ ${ }^{2}$ Surendranath College, 24/2 M. G. Road, Kolkata, West Bengal 700009, India \\ ${ }^{3}$ National Centre for Radio Astrophysics, TIFR, Post Bag 3, \\ Ganeshkhind, Pune 411007, India
}

(Received 23 July 2018; published 29 January 2019)

\begin{abstract}
We study the potential of the Square Kilometre Array in the first phase (SKA1) in detecting dark matter annihilation signals from dwarf spheroidals in the form of diffuse radio synchrotron. Taking the minimal supersymmetric standard model as an illustration, we show that it is possible to detect such signals for dark matter masses about an order of magnitude beyond the reach of the Large Hadron Collider, with about 100 hours of observation with the SKA1.
\end{abstract}

DOI: 10.1103/PhysRevD.99.021302

\section{INTRODUCTION}

The upcoming low-frequency radio telescope, Square Kilometre Array Phase 1 (SKA1), can well surpass the Large Hadron Collider (LHC) reach in unveiling new physics responsible for dark matter (DM). This is shown for the minimal supersymmetric standard model (MSSM), where the lightest neutralino $\left(\chi_{1}^{0}\right)$ is the DM candidate. While the LHC is unlikely to see signatures of supersymmetry (SUSY) for $\left(m_{\chi_{1}^{0}} \gtrsim\right) 1 \mathrm{TeV}$, especially for colored superparticle masses above $\simeq 3 \mathrm{TeV}$ [1,2], DM annihilation in dwarf spheroidal galaxies (dSph) can lead to radio synchrotron emission which clearly rises above the SKA1 detection threshold with 10-100 hours of observation, for $m_{\chi_{1}^{0}}$ up to at least $10 \mathrm{TeV}$. We scan the MSSM parameter space and predict the synchrotron radiation spectra for three such galaxies, for DM annihilation corresponding to the aforesaid SUSY breaking scales. Even with conservative parameter values, the SKA1 should see signals for DM masses one order higher than the reach of the LHC with $\int \mathcal{L} d t=3000 \mathrm{fb}^{-1}[1,2]$.

The SKA1 is expected to address many important questions in astrophysics and cosmology [3]. It has relevance in the physics of elementary particles, too. Foremost in this context is the issue of DM, provided it is constituted of elementary particle(s). While there is no unique candidate theory yet, the MSSM shows a logically

Published by the American Physical Society under the terms of the Creative Commons Attribution 4.0 International license. Further distribution of this work must maintain attribution to the author(s) and the published article's title, journal citation, and DOI. Funded by SCOAP. satisfactory way to obtain a stable neutral particle, especially the lightest neutralino $\left(\chi_{1}^{0}\right)$, which satisfies the requirements of DM. The LHC, however, has not found any signals of it so far, up to colored new particle masses $\gtrsim 2 \mathrm{TeV}$ [4]. On the other hand, spectra in the multi-TeV range can be phenomenologically allowed and satisfy all requirements of $\mathrm{DM}$, if one defers judgements on the somewhat fuzzy issue of naturalness. While the LHC cannot see such heavy superparticles and the fate of any future collider is uncertain, we show below that the SKA1 in its first phase itself can detect diffuse radio synchrotron signals of DM annihilation for such high $m_{\chi_{1}^{0}}$.

\section{SCHEME OF ANALYSIS}

In dSphs and galactic clusters, DM-pairs annihilate into standard model (SM) particles such as $b \bar{b}, t \bar{t}, W^{+} W^{-}$, $\tau^{+} \tau^{-} \ldots$. The subsequent cascades produce copious $e^{+} e^{-}$ pairs whose energy distribution is determined by the source function

$$
Q_{e}(E, r)=\frac{\langle\sigma v\rangle}{2 m_{\chi}^{2}} \rho_{\chi}^{2}(r) \sum_{f} \frac{d N_{f}^{e}(E)}{d E} B_{f},
$$

where $m_{\chi}$ is the DM mass ( $m_{\chi_{1}^{0}}$ in MSSM), $\langle\sigma v\rangle$ and $\rho_{\chi}$ are the DM annihilation rate in any aforementioned channel with fraction $B_{f}$ and DM density inside the Galaxy, respectively. Here we have used the NFW profile for Draco [5,6] and Ursa Major II [7], and the Einasto profile for Segue 1 [8], with the parameters of the profile chosen such that they are consistent with the kinematical observations of these galaxies. $d N_{f}^{e}(E) / d E$ is the energy distribution of the $e^{ \pm}$per annihilation. 
Prediction of the synchrotron signal produced by these $e^{+} e^{-}$pairs requires tracking their propagation through galactic media. Accounting for the energy loss via various electromagnetic processes as well as diffusion, the steady state distribution $d n / d E(E, r)$ of the $e^{ \pm}$as a function of energy $E$ and distance $r$ from the center of the galaxy can be obtained by solving the equation $[6,9]$

$D(E) \nabla^{2}\left(\frac{d n}{d E}\right)+\frac{\partial}{\partial E}\left(b(E) \frac{d n}{d E}\right)+Q_{e}(E, r)=0$,

where $b(E)$ denotes the energy loss due to various radiative processes like the inverse Compton scattering, synchrotron radiation, Coulomb losses, and bremsstrahlung. $D(E)$ is the diffusion parameter which is assumed to have the Kolmogorov form $D=D_{0} E^{\gamma}$ [5-8], $D_{0}$ being the diffusion coefficient. One finally obtains the frequency spectrum of observed photons by folding $d n / d E$ with the synchrotron

TABLE I. Parameters characterizing different classes of benchmarks. $m_{\tilde{l}}$ stands for all three slepton families, except in A1c (see Table II) where $m_{\tau_{1}}$ has been fixed at $=1.03 m_{\chi_{1}^{0}}$ to emphasize the $\tau^{+} \tau^{-}$annihilation channel.

\begin{tabular}{lcccccc}
\hline \hline & $m_{\tilde{l}}$ & $m_{\tilde{Q}_{1,2.3}}$ & $m_{\tilde{q_{R}}}$ & $A_{t}$ & $m_{\tilde{t}_{R}}$ & $m_{\tilde{g}}$ \\
\cline { 2 - 7 } Cases & $(\mathrm{TeV})$ & $(\mathrm{TeV})$ & $(\mathrm{TeV})$ & $(\mathrm{TeV})$ & $(\mathrm{TeV})$ & $(\mathrm{TeV})$ \\
\hline A & $5-5.5$ & 10 & 10 & -2 & 10 & 10 \\
B & $4-4.5$ & 5 & 5 & -4 & 5 & 5 \\
C & $4-4.5$ & 5 & 5 & -4 & 1.95 & 5 \\
D & $5-5.5$ & 10 & 10 & -2 & 2.05 & 10 \\
E & 10 & 10 & 10 & -2 & 10 & 10 \\
F & 15 & 20 & 20 & -1 & 20 & 20 \\
\hline \hline
\end{tabular}

power spectrum, for which a simplified expression is available for frequencies above the gyrofrequency and plasma frequency $[6,9,10]$.

Nearby ultrafaint $\mathrm{dSphs}$ are appropriate for studying such diffuse radio signals, as opposed to the usual galaxies and clusters, as their low star formation rate minimizes the uncertain contribution of astrophysical processes. Their relative proximity (most of them are satellites of the Milky Way) and high DM content, as inferred from the observed mass-to-light ratios within their half-radii, are of further advantage. Some of these dSphs have been observed using existing radio telescopes with the aim of recording such diffuse emission, although no signal has materialized so far $[7,8,11,12]$. The ultrafaint nature of these galaxies necessitates a more sensitive telescope like SKA1 for detecting the radio synchrotron signal [13]. Here we predict the diffuse signal considering the parameters for Draco dSph (mainly because the various relevant parameters like the $J$ factor are better constrained for this object [14]), though even higher flux is expected out of the nearer dSphs such a Segue 1 and Ursa Major II, as shown later in this note.

\section{RESULT AND DISCUSSION}

For a $\chi_{1}^{0}$ DM in MSSM, the observed radio flux [obtained via the velocity averaged quantity $\langle\sigma v\rangle$ (calculated using micrOMEGAs 4.3 .1 [15])] depends on not only $m_{\chi_{1}^{0}}$ but also the particle spectrum and other MSSM parameters that determine the annihilation rates and branching ratios, and also the energy of $e^{ \pm}$transported across the dSph. Some recent works $[6,13,16]$ have treated $m_{\chi}$ and $\langle\sigma v\rangle$ as two free parameters and studied the consequences of different "dominant" annihilation channels. We instead

TABLE II. Parameters in different benchmark models within the classes listed in Table I, and the corresponding DM masses and annihilation channels.

\begin{tabular}{lrrrrrrr}
\hline \hline Model & $M_{1}(\mathrm{GeV})$ & $M_{2}(\mathrm{GeV})$ & $\mu(\mathrm{GeV})$ & $M_{A}(\mathrm{GeV})$ & $\tan \beta$ & Annihilation channel & $m_{\chi_{1}^{0}}(\mathrm{GeV})$ \\
\hline A1a & 1020.0 & 2000.0 & 1180.0 & 2113.0 & 20 & $b \bar{b}(85 \%), \tau^{+} \tau^{-}(14 \%)$ & 1000.6 \\
A1b & 2097.2 & -3536.3 & 1134.8 & 7022.6 & 20 & $W^{+} W^{-}(55 \%), Z^{+} Z^{-}(45 \%)$ & 1163.0 \\
A1c & 1030.0 & 3000.0 & 1150.0 & 2200.0 & 5 & $\tau^{+} \tau^{-}(38 \%), t \bar{t}(37 \%), b \bar{b}(22 \%), W^{+} W^{-}(1.7 \%), Z^{+} Z^{-}(1.3 \%)$ & 1006.7 \\
A2a & 3932.4 & 3645.7 & -3427.5 & 7001.2 & 20 & $b \bar{b}(76 \%), \tau^{+} \tau^{-}(15 \%), t \bar{t}(4 \%), W^{+} W^{-}(3 \%), Z^{+} Z^{-}(2 \%)$ & 3459.4 \\
A2b & 5537.0 & -2976.8 & -3372.3 & 6517.9 & 20 & $W^{+} W^{-}(91 \%), b \bar{b}(7.6 \%), \tau^{+} \tau^{-}(1.4 \%)$ & 3085.4 \\
A2c & 4477.6 & 3977.5 & 4330.9 & 8293.9 & 20 & $b \bar{b}(53.4 \%), W^{+} W^{-}(35 \%), \tau^{+} \tau^{-}(11 \%)$ & 4090.6 \\
A3 & -312.0 & 1000.0 & 400.0 & 690.8 & 10 & $t \bar{t}(79.4 \%), b \bar{b}(16.3 \%), \tau^{+} \tau^{-}(2.2 \%), W^{+} W^{-}(1 \%)$ & 302.0 \\
B1a & -1013.8 & 2022.0 & 1150.0 & 2113.0 & 20 & $b \bar{b}(72 \%), t \bar{t}(16 \%), \tau^{+} \tau^{-}(12 \%)$ & 1000.0 \\
B1b & -3884.7 & 3550.0 & 1132.7 & 3627.7 & 20 & $W^{+} W^{-}(55 \%), Z^{+} Z^{-}(45 \%)$ & 1153.4 \\
B2a & -3485.5 & 4177.9 & 3354.3 & 6820.0 & 20 & $b \bar{b}(76 \%), \tau^{+} \tau^{-}(15 \%), W^{+} W^{-}(3 \%), \bar{t}(3 \%), Z^{+} Z^{-}(2.8 \%)$ & 3368.0 \\
B2b & -3930.2 & -2598.1 & -2957. & 5752.4 & 20 & $W^{+} W^{-}(94.2 \%), b \bar{b}(5 \%)$ & 2662.0 \\
B3 & -295.0 & 1000.0 & 400.0 & 668.0 & 20 & $b \bar{b}(50 \%), t \bar{t}(42 \%), \tau^{+} \tau^{-}(7 \%)$ & 286.0 \\
C & -1012.0 & 3000.0 & 2000.0 & 2033.5 & 10 & $b \bar{b}(63.6 \%), t \bar{t}(26 \%), \tau^{+} \tau^{-}(10.2 \%)$ & 1012.4 \\
D & 1015.0 & 3000.0 & 2000.0 & 2047.0 & 10 & $b \bar{b}(60 \%), t \bar{t}(30 \%), \tau^{+} \tau^{-}(10 \%)$ & 1015.4 \\
E & 8600.0 & 10000.0 & 8500.0 & 17035.0 & 20 & $b \bar{b}(79.1 \%), \tau^{+} \tau^{-}(18.3 \%), t \bar{t}(2.5 \%)$ & 8498.0 \\
F & 11000.0 & 9700.0 & 9965.0 & 20000.0 & 20 & $b \bar{b}(78.5 \%), \tau^{+} \tau^{-}(17.8 \%), t \bar{t}(1.9 \%), W^{+} W^{-}(1.6 \%)$ & 9947.4 \\
\hline \hline
\end{tabular}




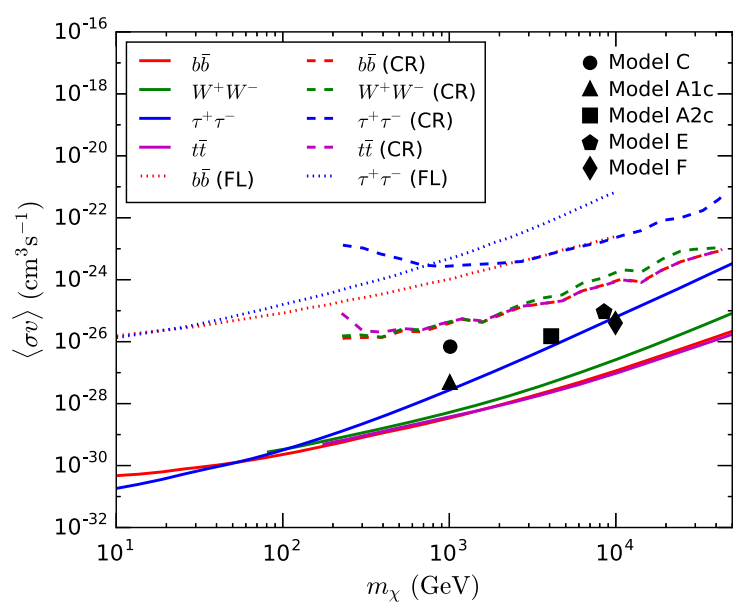

FIG. 1. Lower limits (solid lines) of observability of radio flux from Draco in the $\langle\sigma v\rangle-m_{\chi}\left(m_{\chi_{1}^{0}}\right.$ in MSSM) plane at SKA1 with 100 hours, for various DM annihilation channels. Dashed and dotted lines denote the corresponding 95\% CL upper limits from cosmic-ray (CR) antiproton observation and six years of Fermi LAT (FL) data [25], respectively.

select various MSSM benchmark regions, especially those with heavy superparticles undetectable at the LHC [1,2] and use the full dynamics of the model in terms of the emergent annihilation channels. These benchmarks are listed in Tables I and II. There are four broad classes. (A) has all squarks/gluinos and sleptons well above LHC detection limits, but with a hierarchy between squarks and sleptons. (B) includes somewhat lighter but still undetectable superparticles, but with no hierarchy between colored and colorless ones. (C) and (D) have similar spectra as in (A) and (B) but with lighter top squarks in each case. (E) and (F) correspond to ultrahigh $\chi_{1}^{0}$ masses close to $10 \mathrm{TeV}$. These regions identify DM candidates beyond the commonly conceived domain of naturalness. Further categories within each class reflect different combinations of other MSSM parameters which drive annihilation in different channels. In addition, spectra with $\chi_{1}^{0}$ beyond the LHC detection limit have been juxtaposed with relatively light ones for comparison. All benchmarks satisfy the constraints coming from the collider $[4,17,18]$ and direct DM searches [19], relic density ${ }^{1}$ [20], lightest neutral Higgs mass [21] (calculated at the two-loop level), flavor physics [22], $(g-2)_{\mu}[23]$ etc.

Figure 1 shows the minimum $\langle\sigma v\rangle$ required in various channels for detection with 100 hours (bandwidth = $300 \mathrm{MHz}$ ) at the SKA1, for the dSph Draco. The corresponding annihilation channel has to dominate in each

\footnotetext{
${ }^{1}$ We have demanded that the relic density should lie within stipulated upper and lower limits, as is expected in a singlecomponent DM scenario. The $\langle\sigma v\rangle$ required for relic density calculation needs to be evaluated in a way appropriate for the decoupling temperature, including coannihilation channels.
}

case, for the lower limit to hold. We also indicate the model-independent upper limits on annihilation rates in these channels as functions of the DM particle mass, obtained from cosmic ray antiproton data [24]. The regions bounded by the upper and lower limits represent the area where DM annihilation in this Galaxy can certainly be detected within 100 hours. For Draco, with the NFW profile and a galactic magnetic field $(B)$ of $1.0 \mu G$, $D_{0}=3 \times 10^{28} \mathrm{~cm}^{2} \mathrm{~s}^{-1}$, and $\gamma=0.3$ [5], all of our benchmark points whose samples are shown as black spots (mostly beyond the LHC reach [1,2]) fall in the detectable range. Remarkably, this pushes the radio search limit up to $m_{\chi_{1}^{0}} \sim 8.5 \mathrm{TeV}$. The reach goes up to even $10 \mathrm{TeV}$ if there is substantial annihilation in the $b \bar{b}$ channel.

The frequency spectra of the predicted radio signals are shown in Fig. 2. The expected SKA1 sensitivities in the frequency range $350 \mathrm{MHz}-50 \mathrm{GHz}$ [26] are also shown for observations over 10, 100, and 1000 hours. $^{2}$ Although the curves are drawn using the NFW profile for Draco, we have checked that the predictions remain very similar for other profiles such as Burkert and D05 [5]. Also, we have assumed no halo substructures which can in principle enhance the flux even further [10]. As per current understanding, significant radio signals from astrophysical processes are unlikely, as dSphs are mostly devoid of gas and have almost no intrinsic sources of high energy $e^{ \pm}$. The other possible sources of contamination are the astrophysical foregrounds; however, they too are expected to be subdominant for the SKA1 as the large baselines will help in resolving out these objects. On the whole, detection is almost certain for each case within 100 hours; there are several benchmark points where even ten hours should suffice. Note that the flux depends on $m_{\chi_{1}^{0}},\langle\sigma v\rangle$, and $B_{f}$. Thus, MSSM dynamics crucially decide detectability. Overall, the SKA1 clearly goes beyond the LHC in the SUSY-DM search [1,2]. As Fig. 1 shows, a neutralino DM with a mass on the order of $10 \mathrm{TeV}$ (or perhaps more) may be rendered visible in the process.

While the above results are presented for $B=1.0 \mu G$ (typical of a dSph like Draco where the magnetic field has been measured [27]), the predictions with other values, namely, $B=10.0$ and $0.1 \mu G$, are presented in Fig. 3 (left). We thus see that even for the pessimistic value of $0.1 \mu G$, the signals are detectable up to $10^{3.4}\left(10^{3.8}\right) \mathrm{MHz}$ for 100 (1000) hours of observation. Figure 3 (right) shows the effect of different $D_{0}$. We once more include the "unfavorable" value of $D_{0}=3 \times 10^{29} \mathrm{~cm}^{2} \mathrm{~s}^{-1}, \gamma=0.3$, for which detectability should be rather high in the range

\footnotetext{
${ }^{2}$ It is possible that the SKA1 design may undergo minor changes in the future, leading to gaps in the frequency coverage and revisions in the sensitivity estimates. This should not affect our main conclusion, since the predicted signals are well above the sensitivity limits.
} 

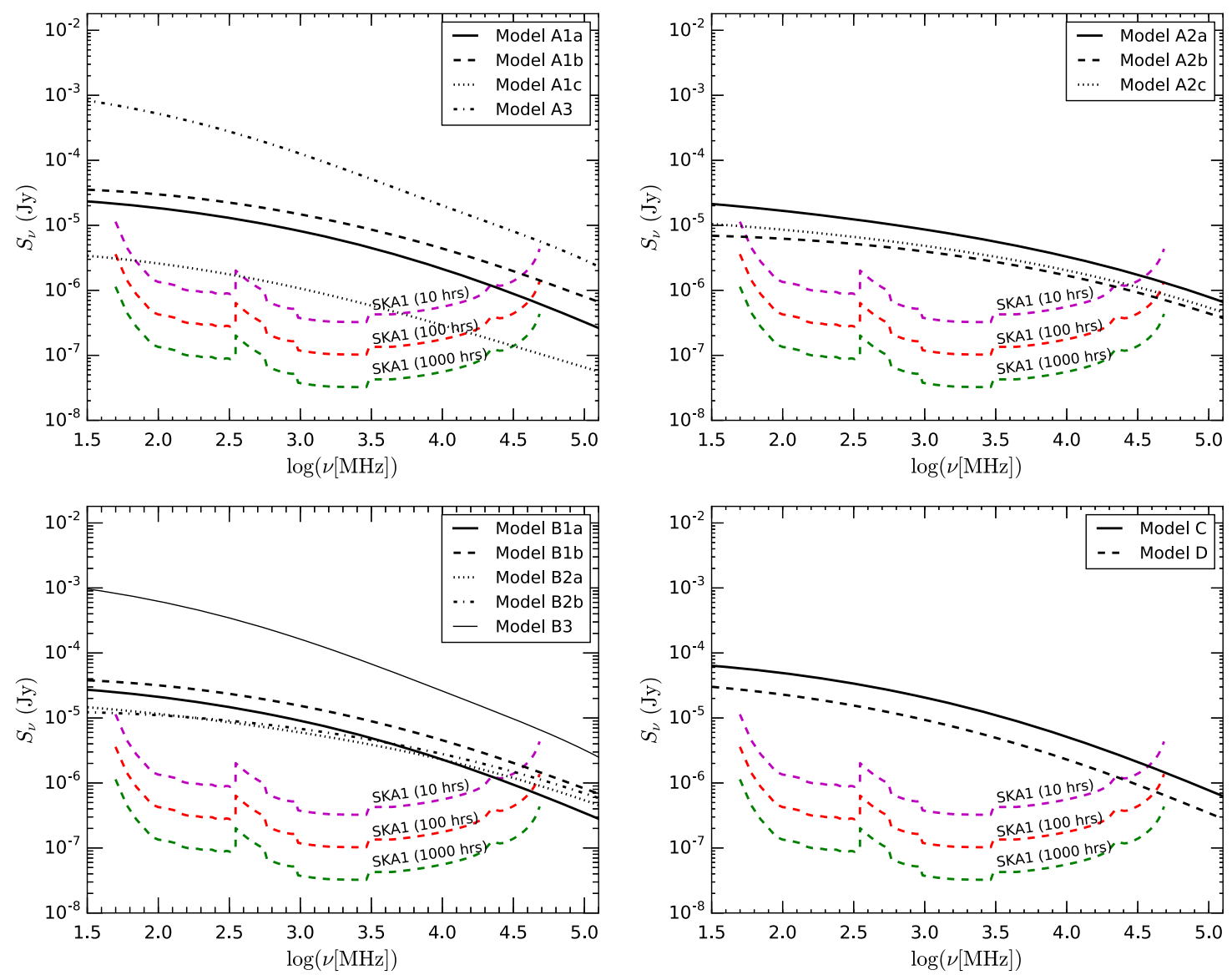

FIG. 2. Synchrotron fluxes for various models (listed in Table I and II) in the Draco dSph galaxy $\left(D_{0}=3 \times 10^{28} \mathrm{~cm}^{2} \mathrm{~s}^{-1}, B=1 \mu G\right)$. The SKA1 sensitivity curves for 10, 100, and $1000 \mathrm{hrs}$ are also shown for bandwidth $=300 \mathrm{MHz}$.

$10^{2-4} \mathrm{MHz}$, for a neutralino mass $\sim 4 \mathrm{TeV}$, with the colored particle masses at $10 \mathrm{TeV}$.

We finally show in Fig. 4 some predictions for galaxies nearer than Draco, namely, Ursa Major II and Segue 1, with $B=1.0 \mu G, D_{0}=3 \times 10^{26} \mathrm{~cm}^{2} \mathrm{~s}^{-1}$, and appropriate values of $\gamma$ [5-8]. Benchmark A1a is used for

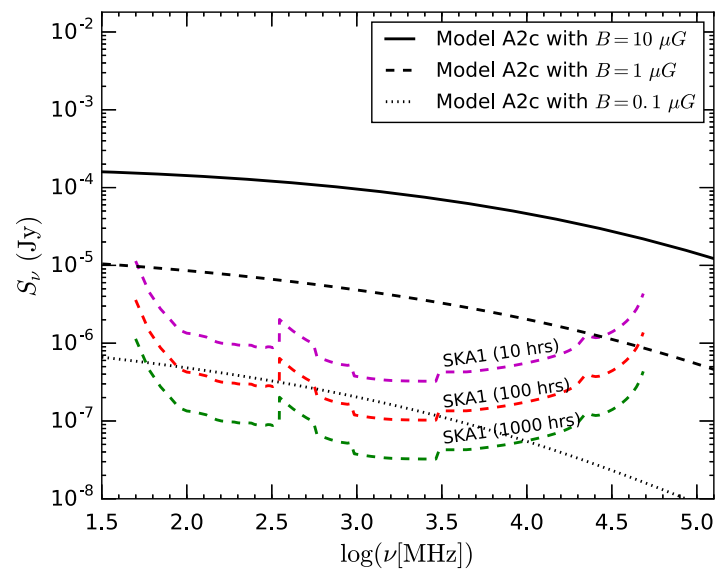

illustration. While detectability is much above the threshold here, a comparison with Draco tells us that Segue 1 and Ursa Major II hold high hopes for DM annihilation detection, even with larger $m_{\chi_{1}^{0}}$. Even if SKA1 succeeds in setting upper limits on the flux for most of our benchmark points, it will be possible to probe and

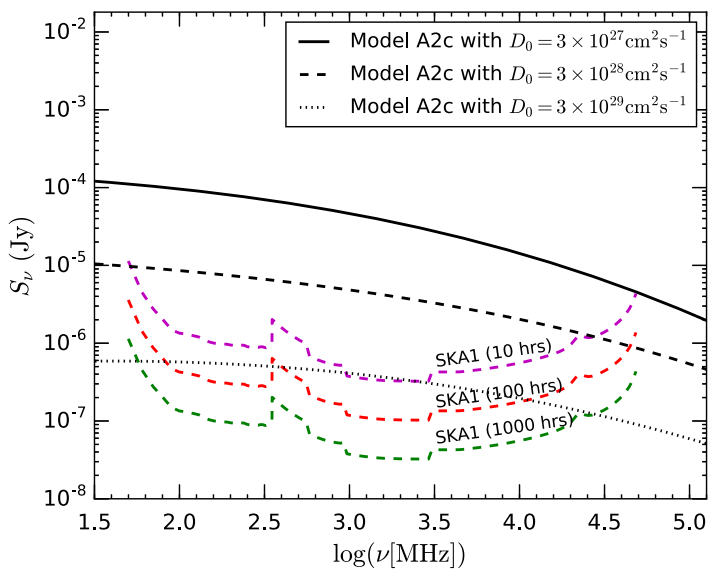

FIG. 3. Left: Synchrotron fluxes for model A2c for Draco $\left(D_{0}=3 \times 10^{28} \mathrm{~cm}^{2} \mathrm{~s}^{-1}\right)$ with various magnetic fields $B=10.0,1.0$, and $0.1 \mu G$. Right: Synchrotron fluxes for model A2c for Draco $(B=1 \mu G)$ with different $D_{0}=3 \times 10^{27}, 3 \times 10^{28}$, and $3 \times 10^{29} \mathrm{~cm}^{2} \mathrm{~s}^{-1}$. 


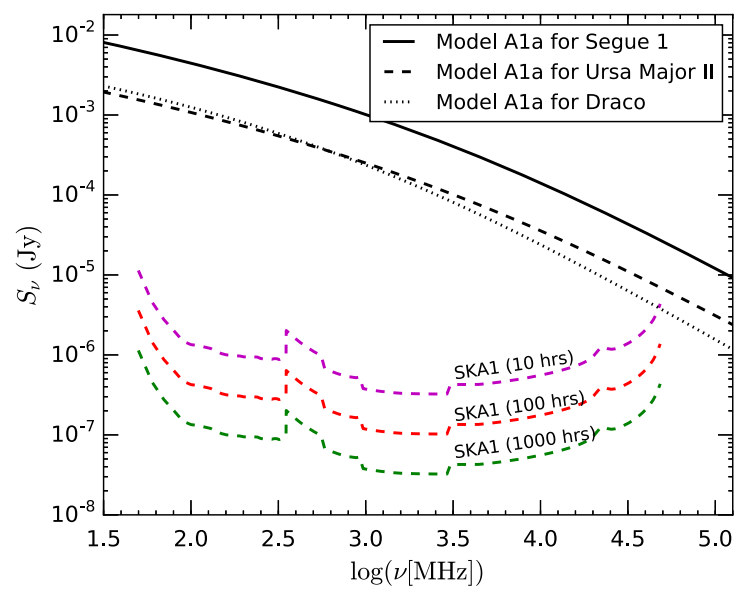

FIG. 4. Comparison of synchrotron fluxes in model A1a among Draco, Ursa Major II and Segue 1 with $D_{0}=3 \times 10^{26}$ and $B=1 \mu G$.

constrain regions of hitherto unexplored regions in the MSSM parameter space well. Observations of the signal in different wavebands, say, radio and $\gamma$-ray frequencies, from any $\mathrm{dSph}$ may enable also us to break the degeneracies between the MSSM parameters and $B, D_{0}$.

Table III shows the annihilation cross sections of the $\chi_{1}^{0} \mathrm{DM}$ for all our benchmark points, for which the corresponding $\chi_{1}^{0}$ masses are supplied in Table II. Side by side, the new particles apart from $\chi_{1}^{0}$, which play the most crucial roles in annihilation are listed, along with their masses for the corresponding benchmarks. As we can see, such role is mostly played among superparticles by the $\chi_{1}^{ \pm}$ and $\chi_{2}^{0}$ on the one hand, and the $\tilde{t_{1}}, \tilde{b_{1}}$, and $\tilde{\tau_{1}}$, on the other. The masses for these particles evidently lie beyond the reach of the LHC for an integrated luminosity of $3000 \mathrm{fb}^{-1}$.

For some benchmark points, the neutral pseudoscalar Higgs $\left(A^{0}\right)$ has an important role. While the corresponding $M_{A}$ in each case is currently allowed by all data, they (with the appropriate values of $\tan \beta$ as in Table II) are unlikely to be seen in any direct signal even at the highluminosity run.

It is true that some representative MSSM parameters values have been included in our sixteen benchmarks. It is of course possible to carry out a similar analysis with a simplified SUSY model containing fewer free parameters. However, we have not restricted ourselves to such scenarios, since our main point is made more emphatically in the general case. The point is that the general MSSM parameter space contains a wide range when not only the $\chi_{1}^{0} D M$ but also the (super)particles responsible for its annihilation within dSph's lie beyond the LHC reach. This is evident form Table III; one is now looking at scenarios with $m_{\chi_{1}^{ \pm}}, m_{\chi_{2}^{0}} \in[1150,4100 \mathrm{GeV}], m_{{\tilde{\tau_{1}}}_{1}} \in[1000,5500 \mathrm{GeV}]$, $m_{\tilde{t}_{1}} \in[1750,5000 \mathrm{GeV}], m_{\tilde{b_{1}}} \simeq 5000 \mathrm{GeV}, M_{A} \in[670$,
TABLE III. Annihilation rates $(\langle\sigma v\rangle)$ for all the benchmark points along with the list of (super)particles dominantly responsible for $\chi_{1}^{0} \mathrm{DM}$ annihilation for any particular benchmark point.

\begin{tabular}{lcc}
\hline \hline & & $\begin{array}{c}\text { BSM } \\
\text { particles dominantly responsible } \\
\text { for annihilation and their } \\
\text { masses }(\mathrm{GeV})\end{array}$ \\
\hline Model & $\left(10^{-26} \mathrm{~cm}^{3} \mathrm{~s}^{-1}\right)$ & $A^{0}(2113.0), \tilde{\tau_{1}}(5497.0)$ \\
A1a & 0.27 & $\tilde{\chi_{1}^{ \pm}}(1163.5), \tilde{\chi_{2}^{0}}(1163.4)$ \\
A1b & 0.77 & $\tilde{\tau_{1}}(1037.0), A^{0}(2200.0)$ \\
A1c & 0.05 & $A^{0}(7001.2), \tilde{\tau_{1}}(5490.0)$ \\
A2a & 1.76 & $\tilde{\chi_{1}^{ \pm}}(3085.5), \tilde{A^{0}}(6517.9)$ \\
A2b & 1.01 & $A^{0}(8293.9), \tilde{\chi_{1}^{ \pm}}(4090.8)$, \\
A2c & 1.47 & $\tilde{\tau_{1}}(5487.2)$ \\
A3 & 1.16 & $A^{0}(690.8)$ \\
B1a & 0.3 & $A^{0}(2113.0), \tilde{b_{1}}(5159.4)$, \\
B1b & 0.79 & $\tilde{t_{1}}(5047.0), \tilde{\tau_{1}}(4496.7)$ \\
B2a & 1.19 & $\tilde{\chi_{1}^{ \pm}}(1153.8), \tilde{\chi_{2}^{0}}(1154.8)$ \\
B2b & 1.3 & $A^{0}(6820.0), \tilde{b_{1}}(5150.6)$, \\
B3 & 1.3 & $\tilde{\tau_{1}}(4488.3), \tilde{t_{1}}(5015.2)$ \\
C & 0.69 & $\tilde{\chi_{1}^{ \pm}}(2662.2), A^{0}(5752.4)$ \\
D & 0.31 & $A^{0}(668.0)$ \\
E & 9.12 & $A^{0}(2033.5), \tilde{t_{1}}(2041.0)$, \\
F & 3.83 & $\tilde{b_{1}}(5085.5), \tilde{\tau_{1}}(4497.3)$ \\
\hline \hline & & $A^{0}(2047.0), \tilde{t_{1}}(1788.0)$, \\
$\tilde{\tau_{1}}(5497.5)$ \\
$A^{0}(17035.0)$ \\
$A^{0}(20000.0)$
\end{tabular}

$20000 \mathrm{GeV}$ ]. All of these (excepting perhaps $M_{A} \simeq$ $670 \mathrm{GeV})$ are beyond the $\mathrm{LHC}$ reach $[1,2,18]$.

\section{SUMMARY}

We thus conclude that the SKA1, mostly with 100 hours of observation, should be able to detect radio synchrotron signals of MSSM DM annihilation, for cases where the superparticle masses are well above the reach of the LHC. Even neutralinoes below a TeV, which the LHC cannot probe due to overwhelming backgrounds, are covered by such observation. This holds even for conservative values of astrophysical parameters and thus underscores a new potential of the SKA.

\section{ACKNOWLEDGMENTS}

We thank AseshKrishna Dutta and Subhadeep Mondal for helpful comments. A. K. and B. M. were partially supported by funding available from the Department of Atomic Energy, Government of India, for the Regional Centre for Accelerator-based Particle Physics (RECAPP), Harish-Chandra Research Institute. T. R. C. and S.M. acknowledge the hospitality of RECAPP. 
[1] L. Shchutska (CMS Collaboration), Nucl. Part. Phys. Proc. 273-275, 656 (2016).

[2] ATLAS Collaboration, CERN Report No. ATL-PHYS-PUB2014-010, 2014, http://cds.cern.ch/record/1735031; CMS Collaboration, CERN Report No. CMS-PAS-FTR-13-014, 2013, https://cds.cern.ch/record/1607141; ATLAS Collaboration, CERN Report No. ATL-PHYS-PUB-2013-011, 2013, http://cds.cern.ch/record/1604505.

[3] R. Braun, T. Bourke, J. A. Green, E. Keane, and J. Wagg, Proc. Sci. AASKA2014 (2015) 174.

[4] A. M. Sirunyan et al. (CMS Collaboration), Phys. Rev. D 96, 032003 (2017); M. Aaboud et al. (ATLAS Collaboration), Phys. Rev. D 96, 112010 (2017).

[5] S. Colafrancesco, S. Profumo, and P. Ullio, Phys. Rev. D 75, 023513 (2007).

[6] A. McDaniel, T. Jeltema, S. Profumo, and E. Storm, J. Cosmol. Astropart. Phys. 09 (2017) 027.

[7] A. Natarajan, J. B. Peterson, T. C. Voytek, K. Spekkens, B. Mason, J. Aguirre, and B. Willman, Phys. Rev. D 88, 083535 (2013).

[8] A. Natarajan, J. E. Aguirre, K. Spekkens, and B. S. Mason, arXiv:1507.03589.

[9] S. Colafrancesco, S. Profumo, and P. Ullio, Astron. Astrophys. 455, 21 (2006).

[10] G. Beck and S. Colafrancesco, J. Cosmol. Astropart. Phys. 05 (2016) 013.

[11] K. Spekkens, B. S. Mason, J. E. Aguirre, and B. Nhan, Astrophys. J. 773, 61 (2013).

[12] M. Regis, L. Richter, and S. Colafrancesco, J. Cosmol. Astropart. Phys. 07 (2017) 025; M. Regis, S. Colafrancesco, S. Profumo, W. J. G. de Blok, M. Massardi, and L. Richter, J. Cosmol. Astropart. Phys. 10 (2014) 016.

[13] S. Colafrancesco, M. Regis, P. Marchegiani, G. Beck, R. Beck, H. Zechlin, A. Lobanov, and D. Horns, Proc. Sci. AASKA2014 (2015) 100.
[14] A. Geringer-Sameth, S. M. Koushiappas, and M. Walker, Astrophys. J. 801, 74 (2015).

[15] G. Belanger, F. Boudjema, A. Pukhov, and A. Semenov, Comput. Phys. Commun. 149, 103 (2002).

[16] G. Beck and S. Colafrancesco, Proc. Sci. HEASA2016 (2017) 010 [arXiv:1704.08029].

[17] M. Aaboud et al. (ATLAS Collaboration), Eur. Phys. J. C 78, 995 (2018); A. M. Sirunyan et al. (CMS Collaboration), J. High Energy Phys. 03 (2018) 160.

[18] M. Aaboud et al. (ATLAS Collaboration), J. High Energy Phys. 01 (2018) 055.

[19] E. Aprile et al. (XENON Collaboration), Phys. Rev. Lett. 119, 181301 (2017); D. S. Akerib et al. (LUX Collaboration), Phys. Rev. Lett. 118, 251302 (2017).

[20] P. A. R. Ade et al. (Planck Collaboration), Astron. Astrophys. 571, A16 (2014); J. Harz, B. Herrmann, M. Klasen, K. Kovarik, and P. Steppeler, Phys. Rev. D 93, 114023 (2016); M. Klasen, K. Kovarik, and P. Steppeler, Phys. Rev. D 94, 095002 (2016).

[21] B. C. Allanach, A. Djouadi, J. L. Kneur, W. Porod, and P. Slavich, J. High Energy Phys. 09 (2004) 044.

[22] R. Aaij et al. (LHCb Collaboration), Phys. Rev. Lett. 118, 191801 (2017); S. Sandilya (Belle II Collaboration), Proc. Sci. CKM2016 (2017) 080.

[23] A. Hoecker and W. J. Marciano, http://pdg.lbl.gov/2018/ reviews/rpp2018-rev-g-2-muon-anom-mag-moment.pdf, 2017.

[24] A. Cuoco, J. Heisig, M. Korsmeier, and M. Krmer, J. High Energy Phys. 04 (2018) 004.

[25] M. Ackermann, A. Albert, B. Anderson et al., Phys. Rev. Lett. 115, 231301 (2015).

[26] R. Braun et al., https://astronomers.skatelescope.org/ wp-content/uploads/2017/10/SKA-TEL-SKO-0000818-01_ SKA1_Science_Perform.pdf, 2017.

[27] K. T. Chyży, M. Weżgowiec, R. Beck, and D. J. Bomans, Astron. Astrophys. 529, 15 (2015); 529, A94 (2011). 